\title{
Paracoccidioidomicose em indios brasileiros da tribo Suruí: estudo clínico-laboratorial de 2 casos
}

\author{
Paracoccidioidomycosis in Amerindian populations of the Brazilian \\ Suruí tribe: a clinical and laboratory study of two cases
}

\author{
Marly H.H. Forjaz, Olga Fischman, Zoilo Pires de Camargo, \\ João Paulo Botelho Vieira Filho e Arnaldo Lopes Colombo
}

\begin{abstract}
Resumo Paracoccidioidomicose é considerada a micose sistêmica endêmica mais prevalente na América Latina. Apesar da maior parte da casuística de paracoccidioidomicose ocorrer entre trabalhadores rurais, há poucos casos documentados de ocorrência dessa micose entre índios brasileiros. São apresentados 2 casos de paracoccidioidomicose em índios Suruí, família linguística Tupi-Mondé, procedentes de Cacoal, Rondônia. Ambos apresentaram sorologia positiva à imunodifusão apenas com antígenos da fase miceliana do P. brasiliensis. Os autores apresentam revisão de literatura sobre a ocorrência dessa micose entre índios brasileiros e discutem a necessidade de futuras investigações buscando caracterizar as diferenças regionais de cepas de P. brasiliensis e seu impacto no diagnóstico sorológico dessa micose.
\end{abstract}

Palavras-chaves: Paracoccidioidomicose. Surui. P. brasiliensis.

Abstract Paracoccidioidomycosis has been considered the most frequent endemic systemic mycosis in Latin America. Although most cases of paracoccidioidomycosis involve rural workers, this systemic fungal disease has been scarcely reported among Amerindian populations from Brazil. We report two cases of paracoccidioidomycosis in Tupi-Mondé Amerindians from Cacoal, state of Rondônia, Brazil. Both cases exhibited positive serological results by a specific immunodiffusion test only when the assay was performed with antigens obtained from the mycelial form of P. brasiliensis. The authors present a literature review of paracoccidioidomycosis in Brazilian Amerindians and discuss the need for further investigations about the impact of the antigenic diversity of P. brasiliensis from different geographic areas on the serological diagnosis of PCM.

Key-words: Paracoccidioidomycosis. Suruí. P. brasiliensis.

A paracoccidioidomicose (Pbmicose) é micose sistêmica de evolução subaguda ou crônica, com predomínio de incidência em adultos do sexo masculino, habitantes ou trabalhadores de zona rural, sendo endêmica em quase todos os estados brasileiros ${ }^{91314}$.

A Pbmicose entre os indígenas da Amazônia brasileira foi reconhecida através de inquéritos epidemiológicos por testes cutâneos e sorológicos a partir de $1978^{15}{ }^{16}$. Recentemente, Coimbra Jr et al conduziram inquérito cutâneo com paracoccidioidina e histoplasmina entre índios das tribos Suruí, Gavião e Zorós, da família lingüística Tupi-Mondé, Amazônia Ocidental, identificando reatores positivos em $43,8 \%, 6,4 \%$ e $14,9 \%$ da população investigada nas respectivas tribos $^{6}$. Alves et al realizaram pesquisa sorológica de paracoccidioidomicose entre índios

Disciplina de Biologia Celular, Departamento de Microbiologia, Imunologia e Parasitologia, Disciplinas de Endocrinologia e de Doenças Infecciosas e Parasitárias, Departamento de Medicina, Escola Paulista de Medicina-Universidade Federal de São Paulo.

Endereço para correspondência: Prof. Arnaldo Lopes Colombo. Disciplina de Doenças Infecciosas e Parasitárias/EPM/UNIFESP.

R. Napoleão de Barros 715/7ªndar, 04023-062 São Paulo, SP. Telefax: 55 11 549-6585/572-6348

Email: lemidipa@ruralsp.com.br

Recebido para publicação em 10/7/98. 
representantes da família lingüística Jê, no centro-oeste brasileiro, encontrando apenas um caso positivo para esta micose pelo teste de imunodifusão dupla (IDD) ${ }^{1}$

Revisão de registros da FUNAI realizada por Coimbra Jr. mostrou que, no período 1983-86, 7 casos de Pbmicose teriam ocorrido entre índios Suruí, todos eles com história pregressa de doença crônica pulmonar tratada como tuberculose, apesar de sucessivos exames de escarro negativos para bacilos álcool-ácidos resistentes. Colombo et al ${ }^{6}$ relataram o primeiro caso de paracoccidioidomicose entre índios no Brasil, com documentação micológica desse diagnóstico, sendo reconhecidos posteriormente, nessa mesma região dos Suruí, outros $2 \operatorname{casos}^{920}$. Os índios Suruí (auto-denominados Pahiter) vivem no Parque de Aripuarâ, sudeste do Estado de Rondônia, Amazônia ocidental, região de floresta úmida tropical (Figura 1). A população de indígenas desta tribo, na época destes relatos, era em torno de 300 indivíduos. Estes índios são caçadores, coletores, possuindo roças de plantio de milho, mandioca e macaxeira.

O conhecimento restrito da ocorrência de micoses sistêmicas entre os índios justifica o interesse na documentação de casos de Pbmicose e outras micoses de envolvimento pulmonar, cujo diagnóstico clínico é muitas vezes confundido com tuberculose.

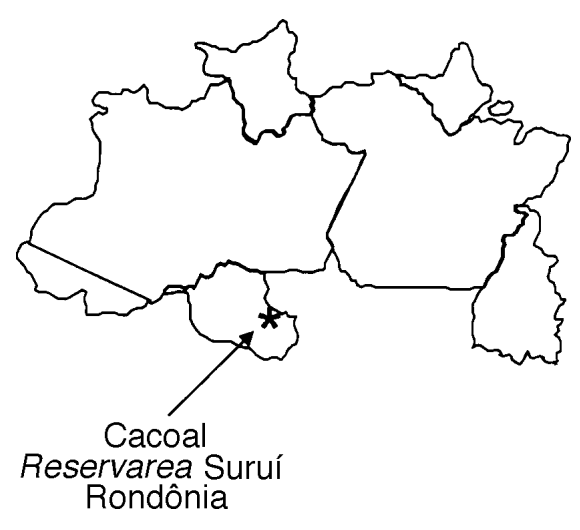

Figura 1 - Localização geográfica da "Reservarea Suruí" na Amazônia Brazileira.

\section{RELATOS DOS CASOS}

Caso 1. AS, masculino, 40 anos, índio Suruí, família lingüística Tupi-Mondé, lavrador, natural e procedente de Cacoal, Rondônia, Amazônia Ocidental. Paciente encaminhado ao Hospital São Paulo com quadro de diminuição de força em MSD e MID há meses. O quadro neurológico instalou-se lenta e progressivamente, acompanhado por emagrecimento e anorexia. Indagado sobre outras queixas, o paciente negava lesão de pele, disfagia, rouquidão ou sintomas respiratórios. Ao exame clínico de entrada apresentava-se consciente, alerta, emagrecido, eupneico, corado e anictérico, com pressão arterial de $90 / 70 \mathrm{mmHg}$, pulso de $84 \mathrm{bpm}$ e temperatura de $37^{\circ} \mathrm{C}$.

Não apresentava lesão de pele, mucosa ou adenomegalia. $O$ exame de tórax e abdomen foi completamente normal. A avaliação neurológica mostrou apenas síndrome de deficit piramidal incompleta de dimídio direito. O exame radiológico de tórax revelou envolvimento pulmonar bilateral, com infiltrado interstícioalveolar. A tomografia de crânio comprovou a existência de lesão expansiva em córtex cerebral esquerdo. $\mathrm{O}$ exame de líquido cefalorraquiano apresentou celularidade e bioquímica normais, com culturas negativas. Foram coletadas 3 amostras de escarro com ausência de BAAR. Paciente foi submetido a exame broncoscópico para elucidação diagnóstica.

Estudo micológico. A cultura do líquido cefalorraquiano para fungos foi negativa. A pesquisa de antígeno específico de Criptococcus neoformans (prova do Látex) também mostrouse negativa. Exame direto a fresco de amostras 
de escarro e lavado brônquico, em preparações microscópicas com a mistura potassa + tinta Quink, Parker (2:1), após conservação do material em câmara úmida a $4^{\circ} \mathrm{C}$ overnigth, revelou raras estruturas globosas com parede refringente, sugestivas de formas solitárias de Paracoccidioides. brasiliensis, mas permitindo dúvida diagnóstica com outras leveduras. A biópsia transbrônquica e o exame citológico do lavado brônquico indicaram processo inflamatório crônico inespecífico e de classe II, respectivamente.

A sorologia pela IDD em agarose suplementada com PEG (polietilenoglicol) 6.000 a $2 \%$ foi positiva, até diluições de 1:64, apenas na presença de paracoccidioidina de fase miceliana (M) do fungo, produzida em meio de cultura preconizado por Negroni, empregando a cepa $\mathrm{Pb}$ B-339, crescida por 30 dias, sob agitação constante de 50rpm, à temperatura ambiente $(11,17)$. Soro controle de paciente caucasiano, da mesma região, com diagnóstico histopatológico de Pbmicose, apresentou o mesmo comportamento em relação às preparações antigênicas de $P$. brasiliensis (Tabela 1).

O paciente foi tratado inicialmente com anfotericina $\mathrm{B}$, apresentando boa melhora clínica. Entretanto, por questões culturais, o índio não concordou em continuar internado no hospital e teve alta a pedido, vindo a falecer após alguns meses.
Caso 2. PS, masculino, 40 anos, índio Suruí, da família linguística Tupi-Mondé, lavrador, natural e procedente de Cacoal, Rondônia. A anamnese, referia tosse com expectoração e dor no peito, às vezes com escarro hemoptoico. Negava disfagia, rouquidão ou lesão de pele. Ao exame clínico de entrada, apresentava-se emagrecido, corado, anictérico, eupneico, com pulso $80 \mathrm{bpm}$, PA $100 / 80 \mathrm{mmHg}$, temperatura $36,5^{\circ} \mathrm{C}$. Não apresentava lesão de pele ou mucosa, sendo notada adenomegalia discreta retroauricular e submandibular. A avaliação pulmonar mostrou a existência de estertores subcreptantes em bases, com ausculta cardíaca normal.

O exame abdominal e avaliação neurológica foram ambos normais. A radiografia de tórax evidenciou infiltrado interstício-alveolar bilateral, com predomínio em campos médios pulmonares. Paciente apresentava ausência de BAAR em amostras de escarro, tendo sido submetido a broncoscopia para avaliação diagnóstica.

Estudo micológico. Amostras de escarro e lavado brônquico, processadas conforme metodologia descrita anteriormente, continham leveduras com vários brotamentos sugestivas de P. brasiliensis. Preparação microscópica de escarro com goma de Berlese + tinta Quink (na proporção 3-4:1) foi selada com verniz de unha e preservada (Figura 2). A biópsia transbrônquica, leveduriformes

Tabela 1 - Diagnóstico sorológico de paracoccidioidomicose em 2 índios e um paciente caucasiano provenientes de Cacoal: comportamento da imunodifusão dupla realizada com antígenos de fase leveduriforme $(L)$ e miceliana $(M)$ do $P$. brasiliensis.

\begin{tabular}{lccc}
\hline Antígeno & Caso 1 (Índio) & Caso 2 (Índio) & Controle (Caucasiano) \\
\hline Leveduriforme & Negativo & Negativo & Negativo \\
Miceliano & $+1: 64$ & $+1: 8$ & $+1: 128$ \\
\hline
\end{tabular}

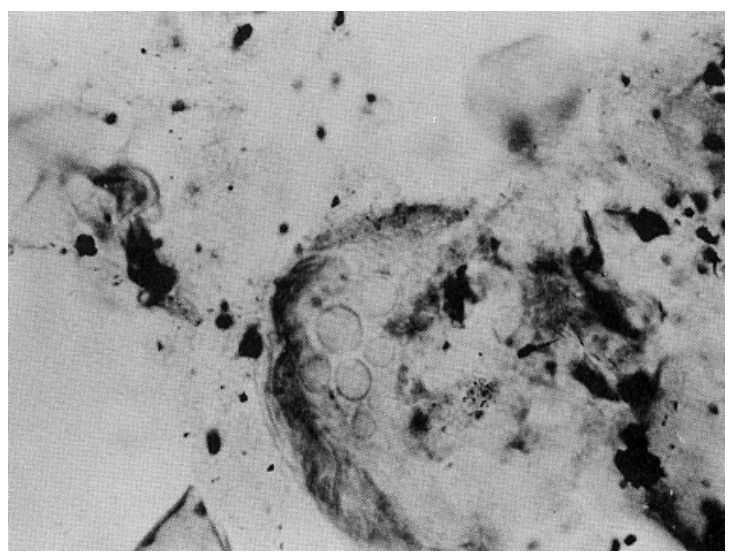

Figura 2 - Preparacão microscópica de escarro com goma de Berlese + tinta Quink (Parker) ilustrando leveduras com múltiplos brotamentos sugestivas de P. brasiliensis. 
típicas de $P$. brasiliensis. A IDD foi positiva até diluição de 1:8, apenas com antígeno de fase miceliana de $P$. brasiliensis impregnada pela prata, revelou células (Tabela 1). A cepa infectante não foi recuperada em cultura. O paciente foi tratado com a combinação de sulfametoxazol-trimetoprima (1600/320mg/dia) obtendo alta em ótimas condições e orientado para uso prolongado da medicação.

\section{DISCUSSÃO}

A Pbmicose é reconhecida como doença importante do México à Argentina, sendo uma das micoses mais estudadas em seus diversos aspectos na América Latina. Entretanto, sua presença entre os índios desse continente é pouco estudada, talvez devido às dificuldades de acesso e comunicação com este grupo, conforme referido por Rolón ${ }^{19}$.

No Brasil, até 1979, inquéritos cutâneos e sorológicos realizados na bacia amazônica não mencionavam reatores positivos entre os índios estudados ${ }^{1516}$. Coimbra et al (1994), conduziram inquérito cutâneo em Rondônia onde detectou vários casos de Pbmicose-infecção entre índios das tribos Suruí (43,8\%), Zoros (14,9\%) e Gavião $(6,4 \%)^{6}$. Este trabalho revelou nova zona endêmica de Pbmicose que se sobrepõe à reservarea Suruí rastreada por Forjaz ${ }^{9}$. Recentemente, inquérito sorológico (imunodifusão-dupla) realizado entre índios da família linguística Jê, revelou um caso de reator positivo ao antígeno de fase leveduriforme de $P$. brasiliensis entre índios Xavantes, reserva de Sangradouro, ilustrando a possibilidade de haver outra reservarea de $P$. brasiliensis nesta área'.

Os programas da FUNAI voltados à assistência médica de população indígena têm propiciado maior contato desta população com hospitais universitários e grupos de pesquisa, estimulando a investigação da ocorrência desta micose entre os índios 157920 .

Os índios Suruí relatados no presente estudo, procedentes de Cacoal (RO), foram encaminhados pela FUNAI ao Hospital São Paulo da Escola Paulista de Medicina e integram a casuística estudada por Forjaz no período novembro/83 maio/88 para o rastreamento de áreas endêmicas e de reservareas de $P$. brasiliensis ${ }^{9}$.

$O$ diagnóstico presuntivo realizado pela equipe médica da FUNAI, em um dos casos relatados (caso 2), foi tuberculose pulmonar, cuja reavaliação clínica e laboratorial realizada no Hospital São Paulo, mostrou tratar-se de Pbmicose pulmonar (6). Este caso ilustra a necessidade de maior suporte técnico nos laboratórios da região, tendo em vista permitir a investigação de micoses entre as causas de infecções crônicas pulmonares destes índios. Esta deficiência de apoio diagnóstico justifica a existência de registros incompletos sobre os casos de Pbmicose na área ${ }^{5}$.

Preparações microscópicas montadas com a mistura $\mathrm{KOH}$ + tinta, combinação de substância digestora-clarificadora-contrastante e corante de material biológico, permitem a visualização da levedura multibrotante característica de $P$. brasiliensis em parasitismo. Brotamentos incipientes são melhor revelados quanto mais tempo o fungo permanecer em contato com a tinta. É metodologia simples, de baixo custo e recomendada para laboratórios que não dispõem de suporte técnico satisfatório, facilitando o reconhecimento do fungo para o diagnóstico correto da micose ${ }^{8}$. Em casos de dúvida, o uso da goma de Berlese + tinta, para a montagem de lâminas semipermanentes, possibilita a remessa da preparação para estudo em centros de Micologia Médica com maior experiência na área.

A pesquisa de anticorpo específico antiP.brasilienis é instumento de auxílio ao diagnóstico bem como ao seguimento clínico para avaliação da resposta terapêutica. Entre as inúmeras técnicas sorológicas disponíveis para o diagnóstico desta doença, a imunodifusão destaca-se pela simplicidade de execução, baixo custo e alta sensibilidade e especificidade ${ }^{2}$. De forma geral, estes ensaios são realizados com antígenos de fase leveduriforme do fungo. Interessante observar que nos casos relatados de Pbmicose em índios Suruí a positividade da imunodifusãodupla foi verificada apenas com a utilização de antígeno de fase miceliana do $P$. brasiliensis. $O$ mesmo fenômeno foi observado com soro de paciente caucasiano proveniente de Cacoal, também com diagnóstico de paracoccidioidomicose.

Vale mencionar que tais soros foram testados, por imunodifusão-dupla, com antígenos de outros fungos, sendo as reações negativas para histoplasmina, esporotriquina, aspergilina e candidina. Conseqüentemente, não se tratava de reações cruzadas com outros agentes de micoses, mesmo porque os títulos encontrados de positividade à fase miceliana de $P$. brasiliensis foram positivos até diluições de 1:8, 1:64 e 1:128, respectivamente, para os 3 casos mencionados. 
Este fato sugere que os 3 pacientes possam ter sido infectados por uma mesma cepa de fungo presente na reservarea Suruí.

A variabilidade antigênica de $P$. brasiliensis tem sido investigada, sendo conhecidas cepas que excretam quantidades diferentes de antígenos responsáveis pela resposta humoral do hospedeiro infectado por este agente, como a glicoproteína de peso molecular $43 \mathrm{kDa}^{16}{ }^{18}$. Este caso aponta para a necessidade de futuras investigações buscando caracterizar as diferenças regionais de cepas de $P$. brasiliensis e seu impacto no diagnóstico sorológico desta micose.

O presente relato documenta a ocorrência de paracoccidioidomicose na Amazônia e alerta os profissionais de saúde que trabalham com populações indígenas sobre a necessidade de considerar as micoses endêmicas entre os diagnósticos diferenciais das infecções pulmonares crônicas que acometem indivíduos desses grupos.

\section{REFERÊNCIAS BIBLIOGRÁFICAS}

1. Alves JR, Colombo AL, Camargo ZP, Vieira Fo JPB. Paracoccidioidomicose e histoplasmose entre índios. Inquérito sorológico pela técnica de imunodifusão em gel de agarose. In: Resumos do IX Congresso Brasileiro de Infectologia, Recife p.87, 1996.

2. Camargo Z, Unterkircher C, Campoy SP, Travassos LR. Production of Paracoccidioides brasiliensis exoantigens for immunodifusion tests. Journal of Clinical Microbiology 26:2147-2151, 1988.

3. Campos MC, Gesztesi JL, Vincentini AP, Lopes JD, Camargo ZP. Expression and isoforms of gp43 in different strains of Paracoccidioides brasiliensis. Journal of Medical and Veterinary Mycology 33:223-227, 1995.

4. Casotto M, Paris S, Camargo ZP. Antigens of diagnostic value in three isolates of Paracoccidioides brasiliensis. Journal of Medical and Veterinary. Mycology 29:243-253, 1991.

5. Coimbra Jr CEA. From shifting cultivation to coffee farming: the impact of change on the health and ecology of the Suruí Indians of the Brazilian Amazon. Tese de Doutoramento, Indiana University, Bloomington, Indiana, USA, 1989.

6. Coimbra Jr CEA, Wanke B, Santos RV, Valle ACF, Costa RLB, Zancopé-Oliveira RM. Paracoccidioidin and histoplasmin sensitivity in Tupí-Mondé Ameridian populations from Brazilian Amazonia. Annals of Tropical Medicine and Parasitology 88:197-207, 1994.

7. Colombo AL, Forjaz MHH, Sader HS, Pereira CAP, Fischman O, Vieira JP. Paracoccidioidomicose autóctone em Suruí, Estado de Rondônia. In: Resumos do XXVI Congresso da Sociedade Brasileira de Medicina Tropical, Natal p.277, 1990.

8. Fischman O, Soni LF, Yonashiro N, Geiger DCP. Consevação de preparações microscópicas a fresco, em líquido clarificador com tinta, em câmara úmida. In: Resumos do XVII Congresso Brasileiro de Microbiologia, Santos p.154, 1993.

9. Forjaz $\mathrm{MHH}$. Estudo da epidemiologia da paracoccidioidomicose. Rastreamento de áreas endêmicas e de "reservareas", no Brasil, através do traçado do perfil migratório-residencial-profissional, de pacientes diagnosticados em São Paulo. Tese de
Doutorado, Escola Paulista de Medicina/UNIFESP, São Paulo, SP, 1989.

10. Forjaz $\mathrm{MHH}$, Camargo ZP, Fischman O, Colombo AL, Candido RC. O índio na cadeia epidemiológica da paracoccidioidomicose. Revista Argentina de Micologia 15:20, 1992.

11. Forjaz $\mathrm{MHH}$, Fischman $\mathrm{O}$, Zaror L, Alchorne AOA, Alchorne MMA. Rapid and easy method for dermatomycosis laboratory diagnosis. In: Resumos do Clinical Dermatology in the year 2000, London p.81, 1990.

12. Forjaz MHH, Fischman O, Zaror L, Yonasuiro N. Agarose gel immunodiffusion tests with the addition of $P E G$ (polyethylenglycol 6000) for the diagnosis and serological follow up of paracoccidioidomycosis infection. Mycopathologia 23:131-133, 1993.

13. Lacaz CS. Micologia Médica. Fungos, actinomicetos e algas de interesse médico. Sarvier, São Paulo, 1994.

14. Londero AT. Epidemiologia. In: Del Negro G, Lacaz CS, Fiorillo AM (eds) Paracoccidioidomicose. Blastomicose sul-americana. Sarvier-EDUSP, São Paulo, p. 85-90, 1982.

15. Mok WY, Ayres CHL, McMillen S. Levantamento sorológico de quatro micoses profundas no Estado do Amazonas, Brasil. Acta Amazonica 9:75-78, 1979.

16. Mok WY, Fava Netto C. Paracoccidioidin and histoplasmin sensitivy in Coari (State of Amazonas), Brazil. American Journal of Tropical Medicine and Hygiene, 27:808-814, 1978.

17. Negroni R. Nuevos estudios sobre antígenos para las pruebas serologicas en la blastomicosis sudamericana. Dermatologia Ibero Latinoamericana 4:409-416, 1968

18. Restrepo A. Sorological comparison of the two morphological phases of Paracoccidioides brasiliensis. Infection and Immunity 2:268-273, 1970.

19. Rolón PA. Paracoccidioidomicosis. Epidemiologia en la República del Paraguay, Centro de Sud América. Mycopathologia 59:67-80, 1976.

20. Valle ACF, Coimbra Júnior CEA, Bornay Linares FI, Monteiro PC, Guimarães MR. Paracoccidioidomicose entre o grupo indígena Suruí de Rondônia, Amazônia, Brasil. Registro de caso. Revista do Instituto de Medicina Tropical de São Paulo 33:407-411, 1991. 